\title{
ARTICLE
}

Acute lymphoblastic leukemia

\section{Mutation, methylation, and gene expression profiles in dup(1q)- positive pediatric B-cell precursor acute lymphoblastic leukemia}

\author{
Rebeqa Gunnarsson ${ }^{1} \cdot$ Sebastian Dilorenzo ${ }^{2,3} \cdot$ Kristina B Lundin-Ström $^{1} \cdot$ Linda Olsson $^{1,4} \cdot$ Andrea Biloglav $^{1}$ • \\ Henrik Lilljebjörn $\mathbb{D}^{1} \cdot$ Marianne Rissler $^{1} \cdot$ Per Wahlberg ${ }^{5} \cdot$ Anders Lundmark $^{5} \cdot$ Anders Castor $^{6} \cdot$ Mikael Behrendtz $^{7}$. \\ Thoas Fioretos ${ }^{1,4} \cdot$ Kajsa Paulsson $\mathbb{1}^{1} \cdot$ Anders Isaksson $\mathbb{1}^{2} \cdot$ Bertil Johansson ${ }^{1,4}$
}

Received: 1 December 2017 / Revised: 19 January 2018 / Accepted: 1 February 2018 / Published online: 12 March 2018

(c) The Author(s) 2018. This article is published with open access

\begin{abstract}
High-throughput sequencing was applied to investigate the mutation/methylation patterns on 1q and gene expression profiles in pediatric B-cell precursor acute lymphoblastic leukemia (BCP ALL) with/without (w/wo) dup(1q). Sequencing of the breakpoint regions and all exons on 1q in seven dup(1q)-positive cases revealed non-synonymous somatic single nucleotide variants (SNVs) in BLZF1, FMN2, KCNT2, LCE1C, NES, and PARP1. Deep sequencing of these in a validation cohort $\mathrm{w}(n=17) /$ wo $(n=94)$ dup(1q) revealed similar SNV frequencies in the two groups (47\% vs. $35 \% ; P=0.42)$. Only $0.6 \%$ of the 36,259 CpGs on 1q were differentially methylated between cases $\mathrm{w}(n=14) /$ wo $(n=13)$ dup(1q). RNA sequencing of high hyperdiploid $(\mathrm{HeH})$ and $\mathrm{t}(1 ; 19)(\mathrm{q} 23 ; \mathrm{p} 13)$-positive cases $\mathrm{w}(n=14) /$ wo $(n=52)$ dup(1q) identified 252 and 424 differentially expressed genes, respectively; only seven overlapped. Of the overexpressed genes in the HeH and $t(1 ; 19)$ groups, 23 and $31 \%$, respectively, mapped to 1q; $60-80 \%$ of these encode nucleic acid/protein binding factors or proteins with catalytic activity. We conclude that the pathogenetically important consequence of dup(1q) in BCP ALL is a genedosage effect, with the deregulated genes differing between genetic subtypes, but involving similar molecular functions, biological processes, and protein classes.
\end{abstract}

Electronic supplementary material The online version of this article (https://doi.org/10.1038/s41375-018-0092-2) contains supplementary material, which is available to authorized users.

Rebeqa Gunnarsson

rebeqa.gunnarsson@med.lu.se

1 Division of Clinical Genetics, Department of Laboratory Medicine, Lund University, Lund, Sweden

2 Array and Analysis Facility, Department of Medical Sciences, Uppsala University, Uppsala, Sweden

3 National Bioinformatics Infrastructure Sweden, Science for Life Laboratory, Uppsala University, Uppsala, Sweden

4 Department of Clinical Genetics and Pathology, Division of Laboratory Medicine, Lund, Sweden

5 Department of Medical Sciences, Molecular Medicine and Science for Life Laboratory, Uppsala University, Uppsala, Sweden

6 Department of Pediatrics, Skåne University Hospital, Lund, Sweden

7 Department of Pediatrics, Linköping University Hospital, Linköping, Sweden

\section{Introduction}

Gain of 1q through a duplication or an unbalanced translocation (both are here denoted "dup(1q)") is found by chromosome banding analysis in $\sim 5 \%$ of pediatric B-cell precursor acute lymphoblastic leukemia (BCP ALL) cases $[1,2]$, but the frequency increases quite substantially if single nucleotide polymorphism array (SNP-A) analyses are performed[3, 4]. The incidence of dup(1q) varies according to genetic subtype, with 1q gain being particularly common in cases with $\mathrm{t}(1 ; 19)(\mathrm{q} 23 ; \mathrm{p} 13) / T C F 3-P B X 1$ fusion $(\sim 60 \%)$, most often as a consequence of the unbalanced der(19)t $(1 ; 19)$, or with high hyperdiploidy $(\mathrm{HeH} ; 20 \%)$; more rarely, dup(1q) is present in $\mathrm{t}(12 ; 21)(\mathrm{p} 13 ; \mathrm{q} 22) / E T V 6-$ RUNX1-positive or B-other cases[2, 4-7]. However, gain of $1 \mathrm{q}$ is not restricted to BCP ALL. In fact, $\operatorname{dup}(1 \mathrm{q})$ is one of the most common genomic imbalances in human neoplasia, such as multiple myeloma, various types of B-cell lymphoma, carcinomas of the breast, colon, and lung, malignant melanoma, and Wilms' tumor[2, 8-15]. The high frequency of 1q gain strongly suggests that it plays an important pathogenetic role in tumorigenesis in general, perhaps by conferring a proliferative advantage, as indicated by a study 
of growth patterns of dup(1q)-positive and -negative chronic lymphocytic leukemia clones in nude mice[16]. Such a proliferative advantage is a possible explanation for the worse prognosis of Wilms' tumors and multiple myelomas with 1q gains[8, 17, 18].

Genomic gains are generally associated with genedosage effects resulting in overexpression of some of the duplicated/amplified genes[19, 20]. However, considering the high frequency of $1 \mathrm{q}$ gain in neoplasia, surprisingly few studies have investigated its effect on gene expression or ascertained pathogenetically important target genes within the duplicated segments. In hematologic malignancies, gain of $1 \mathrm{q}$ has been associated with overexpression of the $C K S 1 B$ gene in 1q21 in multiple myeloma $[17,18]$ and with upregulation of B4GALT3 (1q23), DAP3 (1q22), RGS16 (1q25), TMEM183A (1q32), and UCK2 (1q24) in a few dup (1q)-positive $\mathrm{HeH}$ cases[21]. This notwithstanding, a genedosage effect may not be the only functional consequence of $\operatorname{dup}(1 \mathrm{q})$ - it may also be associated with DNA methylation changes and/or gene mutations on 1q, but this has not been addressed in previous studies of dup(1q)-positive malignancies.

To ascertain the functional consequences of $\operatorname{dup}(1 \mathrm{q})$ in pediatric BCP ALL, we compared, using several types of next-generation sequencing, the mutation and methylation patterns on $1 \mathrm{q}$ and global gene expression profiles between cases with/without (w/wo) 1q gain.

\section{Materials and methods}

\section{Patients}

Twenty-seven dup(1q)-positive, all identified by SNP-A analysis, and 132 dup(1q)-negative pediatric BCP ALL cases were investigated (Supplementary Tables 1 and 2). Based on the SNP-A data, the smallest dup(1q)-positive clone included had a frequency of $\sim 25 \%$ (case 6; Supplementary Tables 1). A flowchart outlining the number of cases included in the various analyses is presented in Supplementary Figure 1.

The 27 cases with 1q gain consisted of $16 \mathrm{HeH}$, eight $\mathrm{t}$ $(1 ; 19)$, one $\mathrm{t}(12 ; 21)$, and two B-other cases. Targeted deep sequencing of $1 \mathrm{q}$ was performed on seven cases, Truseq custom amplicon (TCA) analysis on 17, bisulfite sequencing (BS-Seq) on 15, and RNA sequencing (RNA-Seq) on 14 cases (Supplementary Table 1). The 132 dup(1q)-negative cases, used as a validation or reference cohort in the TCA $(n=94)$, BS-Seq $(n=15)$, and RNA-Seq $(n=52)$ analyses, comprised $51 \mathrm{HeH}$, six $\mathrm{t}(1 ; 19), 32 \mathrm{t}(12 ; 21)$, seven 11q23-rearranged, four $\mathrm{t}(9 ; 22)(\mathrm{q} 34 ; \mathrm{q} 11)$, one low hypodiploid, and 31 B-other cases (Supplementary Table 2). The cases with $\mathrm{t}(1 ; 19), \mathrm{t}(9 ; 22)$, and $\mathrm{t}(12 ; 21)$ were all confirmed to be positive for the TCF3-PBX1, BCR-ABL1, and ETV6$R U N X 1$ fusions, respectively, by fluorescence in situ hybridization (FISH) or reverse-transcription polymerase chain reaction analysis, whereas the cases with $11 \mathrm{q} 23$ rearrangements were all confirmed to be $K M T 2 A$-rearranged by FISH or Southern blot analysis.

The study was approved by the Research Ethics Committee of Lund University (DNR 2016/18) and informed consent for the analyses was obtained according to the Declaration of Helsinki.

\section{Single nucleotide polymorphism array analysis}

The SNP-A analyses and original SNP data have previously been reported[4, 22]. The SNP-A systems HumanOmni1-Quad or Human1M-Duo (Illumina, San Diego, CA, USA), covering $>1,000,000$ SNPs, were used. The analyses were performed according to the manufacturer's instructions and the B-allele frequencies and the $\log 2$ ratios were ascertained by the Genome studio v2011.1 software (Illumina), extracting probe positions from the GRCh37 genome build.

\section{Targeted deep sequencing of $1 \mathrm{q}$}

The SureDesign tool was applied to select probes for the DNA library preparation kit (Agilent Technologies, Santa Clara, CA, USA). Two probe sets were used: (1) probes covering the $1 \mathrm{q}$ breakpoint regions at chr1:142,535,430-

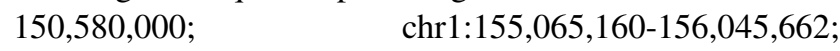
chr1:164,619,900-164,875,964; chr1:186,307,000-

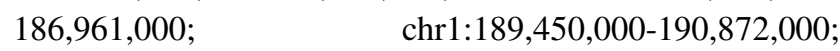
chr1:197,566,843-197,843,204; chr1:215,136,000-

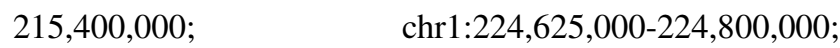
chr1:226,000,000-226,055,000; and chr1:249,000,000$249,250,621$; and (2) probes covering all exons on 1q. This resulted in 220,160 probes with a total size of $13.755 \mathrm{Mb}$.

Seven paired diagnostic/remission samples were included in the library preparation and sequenced on HiSeq 2000 (Illumina) at SciLifeLab, Uppsala, Sweden. The Burrows-Wheeler aligner was used for alignment of the DNA sequences to the human reference genome ( $\mathrm{Hg} 19)$ [23]. Variant calling was performed using MuTect/1.1.5 [24], candidate mutations were annotated with Annovar [25], and structural variant calling for breakpoint mapping was performed with LUMPY/0.2.13[26]. The results from LUMPY were processed with SVTYPER (https://github. com/hall-lab/svtyper) [27] to generate descriptive genotype information, and custom scripts were used to identify candidate somatic structural variants. The filtering criteria used for candidate detection were $\mathrm{A} 0>4$ in the leukemic samples and $\mathrm{A} 0=0$ in the matched remission sample for each variant. 


\section{Truseq custom amplicon analysis}

Based on the single nucleotide variants (SNVs) identified by targeted deep sequencing of 1q, the BLZF1 (basic leucine zipper nuclear factor 1 at 1q24.2), FMN2 (formin 2; 1q43), KCNT2 (potassium sodium-activated channel subfamily $\mathrm{T}$ member 2; 1q31.3), LCE1C (late cornified envelope 1C; 1q21.3), NES (nestin; 1q23.1), and PARP1 (poly(ADP-ribose) polymerase $1 ; 1 \mathrm{q} 42.12$ ) genes were selected for TCA analysis of a larger patient cohort w/wo 1q gains. Probes targeting all exons within these genes were constructed with DesignStudio (Illumina). In total, 111 diagnostic samples $\mathrm{w}(n=17) /$ wo $(n=94) \operatorname{dup}(1 \mathrm{q})$ and 79 paired remission samples from cases $\mathrm{w}(n=10) /$ wo $(n=$ 69) $\operatorname{dup}(1 \mathrm{q})$ at diagnosis were analyzed. The sequence libraries were constructed according to the manufacturer's instructions and the pooled libraries were sequenced on Nextseq 500 (Illumina). Variant calling was performed with MuTect/1.1.7[24]. In the variant calling of the 32 cases without a remission sample, a reference panel was utilized, created from the available 79 remission samples by calling variants using MuTect/1.1.7, and then merging the variants using CombineVariants from GATK/3.4.0[28-30]. Subsequently, the reference panel was used to identify putative somatic variation in leukemia samples lacking a remission sample. All variants were annotated with Annovar[25].

\section{Sanger sequencing}

For the SNVs selected for verification by Sanger sequencing, forward and reverse primers (Supplementary Table 3) were designed with Primer3 (http://bioinfo.ut.ee/primer3/) and purchased from Termo Fisher Scientific (Waltham, MA, US). ChromasLite 2.6 (Technelysium, South Brisbane, Australia) was used for sequence analysis.

\section{Bisulfite sequencing}

Thirty cases $\mathrm{w}(n=15) /$ wo $(n=15) 1 \mathrm{q}$ gains were bisulfite sequenced. The SureSelect Human Methyl-Seq (Agilent), which targets cancer tissue-specific differentially methylated regions, promoters, $\mathrm{CpG}$ islands, shores, and shelves, was used. The library preparations were performed according to the manufacturer's instructions and the pooled libraries were sequenced on HiSeq 2000 (Illumina) at SciLifeLab. The BS-Seq data were processed through a bioinformatic pipeline developed at SciLifeLab (Supplementary Figure 2). Reference genome ucsc.hg19.fasta (bundle 2.8) was processed with Bismark/0.12.2 to create a suitable human reference genome for alignment of bisulfitetreated reads[31]. Analysis of differentially methylated CpGs was performed using the $\mathrm{R}$ package methylKit/0.9.2 and R/3.0-3.2.3[32]. Methylation calls for the cases w/wo dup(1q) were compared for overlapping sites, with each positions coverage required to be in the interval $2 \times-50 \times$. Principal component and hierarchical clustering analyses (PCA and HCA) were performed on $\mathrm{CpGs}$ with differential methylation, as ascertained by methylKit/0.9.2. For a site to be considered differentially methylated between the groups w/wo dup(1q), a difference of $\geq 25 \%$ and a $q$-value less than 0.01 were required. Annotations were downloaded from UCSC (assembly hg19) and matched to gene regions, CpG islands, shores, and shelves in the differential methylation data set. Annotation plots of differential methylation in genomic and $\mathrm{CpG}$ regions, including both hyper- and hypomethylated sites, were created with methylKit (https:// github.com/al2na/methylKit)[32].

\section{RNA sequencing and gene expression profiling}

The RNA-Seq analyses, focusing on fusion genes in 195 pediatric BCP ALL cases, have previously been reported [33]. The RNA-seq data are deposited at the European Genome-phenome Archive under the accession code EGAS00001001795. Qlucore Omics Explorer 3.1 (Qlucore $\mathrm{AB}$, Lund, Sweden) was used for gene expression profiling of $\mathrm{HeH}$ cases $\mathrm{w}(n=7) /$ wo $(n=46)$ and $\mathrm{t}(1 ; 19)$-positive cases $\mathrm{w}(n=7) /$ wo $(n=6) 1 \mathrm{q}$ gain. Cases w/wo dup $(1 \mathrm{q})$ were compared with the paired $t$-test, and a $P$-value of $\leq 0.01$ was used as a cut-off for differentially expressed genes. HCA was performed on the differentially expressed genes in the dup(1q)-positive and -negative $\mathrm{HeH}$ and $\mathrm{t}(1 ; 19)$ groups. For gene ontology (GO) analysis of the differentially expressed genes, the Panther version 12.0 (http://pa ntherdb.org/) was used to extract information on their molecular functions, biological processes, and protein classes.

\section{Results}

\section{Dup(1q) in BCP ALL: near-centromeric breakpoints and whole arm gains}

SNP-A analyses of the 27 dup(1q)-positive cases revealed that the $1 \mathrm{q}$ gains ranged from $24.4 \mathrm{Mb}$ to $103.9 \mathrm{Mb}$, with a median size of $97.9 \mathrm{Mb}$ (Supplementary Table 4). One case (\#3) had two separate $24.4 \mathrm{Mb}$ and $39.3 \mathrm{Mb}$ duplications, whereas all other cases harbored a single, continuous duplication. The minimal overlapping gain in the $\mathrm{HeH}$ subgroup was the $24.4 \mathrm{Mb}$ duplication in case 3 , and in the $\mathrm{t}$ $(1 ; 19)$ subgroup, the smallest overlapping region was a $84.4 \mathrm{Mb}$ duplication in case 16 . In $16(59 \%)$ of the 27 cases and in $13(81 \%)$ of the $16 \mathrm{HeH}$ cases the proximal breakpoints were near-centromeric, occurring in, or proximal to, 1q21.1 (the repetitive nature of the DNA sequence in this 
region made it impossible exactly to map the breakpoints by SNP-A). In $22(81 \%)$ of the cases, the gains included the telomeric region, i.e., the entire 1q arm distal to the proximal breakpoint was duplicated (Supplementary Figure 3 and Supplementary Tables 1 and 4). In cases 2, 3, 4, and 7 with proximal breakpoints distal to $1 \mathrm{q} 21.1$ and/or with distal breakpoints proximal to the telomeric region, the sequence analysis revealed that the breakpoint regions were surrounded by small structural variants that most often were classified as deletions. However, it was not possible to identify the precise locations of the breakpoints.

\section{Similar somatic mutation frequencies on $\mathbf{1 q}$ in cases with/without dup(1q)}

Targeted deep sequencing of the breakpoint regions and of all exons (including also intronic flanking sequences) on 1q revealed a total of 231 somatic SNVs in the seven cases analyzed (range, 18 to 74 SNVs per case; Supplementary Table 5). The majority of the SNVs were intergenic $(151 / 231 ; 65 \%)$ and most were novel $(144 / 231 ; 62 \%)$, i.e., not included in dbSNP (https://www.ncbi.nlm.nih.gov/ projects/SNP/). Of the 80 intragenic SNVs, 61 (76\%) occurred within introns, nine (11\%) were located upstream, downstream, or in the $3^{\prime}$ untranslated regions of genes, and ten $(12 \%)$ were exonic. Of the ten exonic SNVs, three were synonymous and seven non-synonymous. Six of the latter, occurring in the BLZF1, FMN2, KCNT2, LCE1C, NES, and $P A R P 1$ genes, were detected in $17-66 \%$ of the reads and were all verified by Sanger sequencing (Supplementary Table 5). One non-synonymous SNV in the OR2T34 gene (olfactory receptor family 2 subfamily $\mathrm{T}$ member 34 at 1q44) was discarded because it was only present in $4 / 42$ $(10 \%)$ reads.

The TCA analysis of the BLZF1, FMN2, KCNT2, LCE1C, $N E S$, and PARPI genes in the validation cohort $(n=111)$ revealed SNVs in $\geq 1$ of these genes in $76 \%$ (13/17) of the dup(1q)-positive and in 66\% (62/94) of the dup(1q)-negative cases $(P=0.42$; two-tailed Fisher's exact test) (Supplementary Table 6). When only considering non-synonymous SNVs, the corresponding frequencies were $47 \%(8 / 17)$ and $35 \%$ (33/94), respectively $(P=0.42)$.

\section{Similar methylation patterns on $\mathbf{1 q}$ in cases with/ without dup(1q)}

Of the 30 cases analyzed by BS-Seq, three did not pass the quality score test. Hence, the methylation patterns on 1q could be ascertained in 27 cases. HCA and PCA of the cases $\mathrm{w}(n=14) /$ wo $(n=13)$ gain of 1q did not separate these two groups (Supplementary Figure 4). Furthermore, only $201(0.6 \%)$ of the $36,259 \mathrm{CpG}$ sites on 1q were differentially methylated, with 137 (68\%) being hypermethylated and 64 (32\%) hypomethylated. The distributions of differentially methylated $\mathrm{CpGs}$ in promoters, introns, exons, and intergenic regions did not differ between hyper- and hypomethylated CpGs (Supplementary Figure 5).

Comparing the methylation and gene expression data in cases analyzed by both BS-Seq and RNA-Seq, only seven genes displayed both differential methylation of $\mathrm{CpGs}$ and differential expression: RORC was hypermethylated and underexpressed, whereas the GALNT2, PSMB4, SHC1, $S O X 13, S S R 2$, and UCK2 genes were hypomethylated and overexpressed in the dup(1q)-positive cases.

\section{Different deregulated genes in dup(1q)-positive $\mathrm{HeH}$ and $t(1 ; 19)$ cases}

HCA of the global gene expression profiles as well as of the expression patterns of only genes mapping to $1 \mathrm{q}$ in the $\mathrm{t}$ $(1 ; 19)$ cases w/wo 1q gain revealed two cluster groups characterized by the presence or absence of $\operatorname{dup}(1 \mathrm{q})(P \leq 0.01)$, with only one dup(1q)-positive case being grouped together with the non-dup(1q) cases (Supplementary Figure $6 a$ and $b$ ). HCA of the global gene expression profiles in the $\mathrm{HeH}$ cases also clustered most dup (1q)-positive cases together (Supplementary Figure 6c); such a clustering was not found by HCA of the expression of genes only on 1q in the $\mathrm{HeH}$ cases (Supplementary Figure 6d).

In the $\mathrm{HeH}$ group, 252 genes were differentially expressed between cases w/wo $\operatorname{dup}(1 \mathrm{q}) ; 196(78 \%)$ were overexpressed and 56 (22\%) underexpressed (Fig. 1a; Supplementary Table 7). A total of 424 genes were differentially expressed between the $t(1 ; 19)$ cases w/wo dup $(1 \mathrm{q})$, of which $242(57 \%)$ were overexpressed and $182(43 \%)$ underexpressed (Fig. 1b; Supplementary Table 8). In the cases with der(19)t $(1 ; 19)$, resulting in loss of distal 19p, 30 genes located on chromosome 19 were downregulated (Fig. 1b); 16 (53\%) of these mapped to the $1.6 \mathrm{Mb}$ region telomeric to the $T C F 3$ gene at $19 \mathrm{p} 13.3$, a region comprising only $2.8 \%$ of chromosome 19 . Of the 252 and 424 differentially expressed genes in the $\mathrm{HeH}$ and $\mathrm{t}(1 ; 19)$ groups, respectively, only seven overlapped, of which ELK4 (1q32.1), GAS5 (1q25.1), NENF (1q32.3), and SNRPE (1q32.1) were overexpressed and CAVI (7q31.2), POU2AF1 (11q23.1), and ZNF831 (20q13.32) downregulated in dup $(1 \mathrm{q})$-positive cases.

Among the overexpressed genes in the dup(1q)-positive $\mathrm{HeH}$ and $\mathrm{t}(1 ; 19)$ groups, 23\% (46/196) and 31\% (76/242), respectively, were located on 1q (Fig. 1a, b); in comparison, $1,115(4.8 \%)$ of the 23,285 genes included in the analysis were located on 1q. Using Panther for GO analysis, defined molecular functions, biological processes, and protein classes were available for 28,41 , and 37 of the upregulated genes on 1q in the $\mathrm{HeH}$ cases and for 44,74 , and 46 of the 
Fig. 1 Frequencies of significantly $(P<0.01)$ differentially expressed genes (red denotes upregulated genes whereas green denotes downregulated genes) and their chromosomal locations in dup (1q)-positive pediatric $\mathrm{HeH}$ and $t(1 ; 19)$ cases. a Gene expression profiling (GEP) analysis of $\mathrm{HeH}$ cases $\mathrm{w}(n=7) /$ wo $(n=46)$ gain of $1 \mathrm{q}$ revealed that 252 genes were differentially expressed between cases w/wo $\operatorname{dup}(1 \mathrm{q}): 196(78 \%)$ were overexpressed (46 (23\%) of which mapped to 1q) and 56 (22\%) were underexpressed. b GEP analysis of $\mathrm{t}(1 ; 19)$ cases $\mathrm{w}$ $(n=7) /$ wo $(n=7)$ gain of $1 \mathrm{q}$ showed that 424 genes were differentially expressed between the $t(1 ; 19)$ cases w/wo $\operatorname{dup}(1 \mathrm{q})$ : $242(57 \%)$ were overexpressed (76 $(31 \%)$ of which mapped to 1q) and $182(43 \%)$ were underexpressed. In the cases with $\operatorname{der}(19) \mathrm{t}(1 ; 19)$, resulting in loss of distal 19p, 30 genes located on chromosome 19 were downregulated; 16 (53\%) of these mapped to the $1.6 \mathrm{Mb}$ region telomeric to the TCF3 gene at $19 \mathrm{p} 13.3$
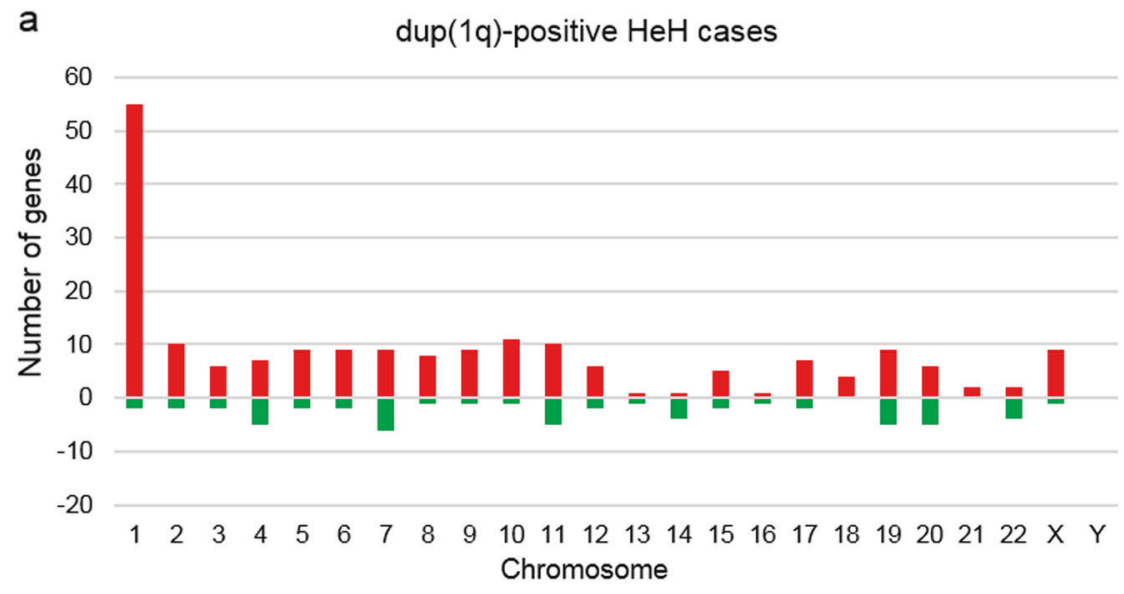

b dup(1q)-positive $t(1 ; 19)$ cases

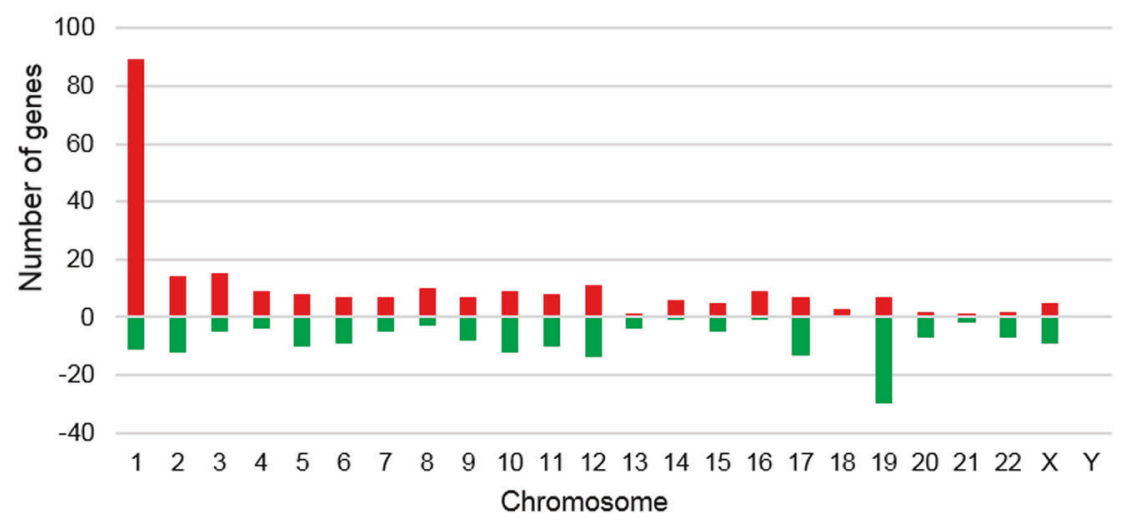

overexpressed $1 \mathrm{q}$ genes in the $\mathrm{t}(1 ; 19)$ cases. In both the $\mathrm{HeH}$ and $\mathrm{t}(1 ; 19)$ groups, the most common molecular functions of the upregulated 1q genes were "binding" (21\% and $43 \%$, respectively), comprising nucleic acid binding and protein binding, and "catalytic activity" (39\% and 36\%, respectively), e.g., hydrolase and transferase activity (Fig. 2a, b). With regard to biological processes of the overexpressed genes in the dup(1q)-positive $\mathrm{HeH}$ and $\mathrm{t}$ $(1 ; 19)$ cases, cellular and metabolic processes, such as cell communication and primary metabolic activity, were the most frequent (56\% and 52\%, respectively) (Supplementary Figure $7 \mathrm{a}$ and b). Eighteen protein classes were represented among the overexpressed genes, of which 13 were common to both the $\mathrm{HeH}$ and $\mathrm{t}(1 ; 19)$ groups; the most frequent were receptors/signaling molecules, transcription factors, and nucleic acid binding proteins (Supplementary Figure 8a and $b)$.

\section{Discussion}

The present study - the first to apply several types of nextgeneration sequencing to ascertain genomic and epigenetic features of dup(1q) in BCP ALL-revealed clustering of near-centromeric breakpoints and gain of the entire chromosome arm in the majority of cases, similar gene mutation frequencies and methylation patterns on $1 \mathrm{q}$ in cases w/wo gain of 1q, and distinct gene expression profiles of $\mathrm{HeH}$ and $\mathrm{t}(1 ; 19)$ cases with $\operatorname{dup}(1 \mathrm{q})$.

Previous studies of solid tumors as well as of some hematologic malignancies, using chromosome banding, FISH, or SNP-A analyses[12, 13, 21, 34-40], have reported that the proximal breakpoints of dup(1q) in most cases are near-centromeric, often within the satellite II (sat II) domain. In the present study, we identified breakpoints in, or proximal to, $1 \mathrm{q} 21.1$ in $\sim 60 \%$ of all cases and in $\sim 80 \%$ of the $\mathrm{HeH}$ cases. This agrees well with prior analyses of $1 \mathrm{q}$ gains in other types of neoplasia. However, in contrast to chromosome banding studies suggesting that the distal breakpoints in $\operatorname{dup}(1 \mathrm{q})$ map proximal to the telomeric $1 \mathrm{q} 44$ band in $~ 75 \%$ of pediatric BCP ALL cases with gain of 1q[2], our SNP-A analyses revealed that the entire chromosome arm was gained in more than $80 \%$ of cases (Supplementary Figure 3 ). This clearly shows that chromosome banding analysis underestimates the sizes of the duplicated segments; thus, that method cannot be used reliably to delineate the minimally gained 1q region. 
Fig. 2 Molecular function ontology classes of genes on $1 \mathrm{q}$ upregulated in dup(1q)-positive cases. a In the $\mathrm{HeH}$ cases, 28 of the 46 upregulated genes on $1 \mathrm{q}$ had defined molecular functions, comprising seven classes. b In the $(1 ; 19)$ cases, 44 of the 76 overexpressed genes on $1 \mathrm{q}$ had defined molecular functions, comprising five ontology classes. Antioxidant activity (GO:0016209); binding (GO:0005488); catalytic activity (GO:0003824); receptor activity (GO:0004872); signal transducer activity (GO:0004871); structural molecule activity (GO:0005198); and transporter activity (GO:0005215) a

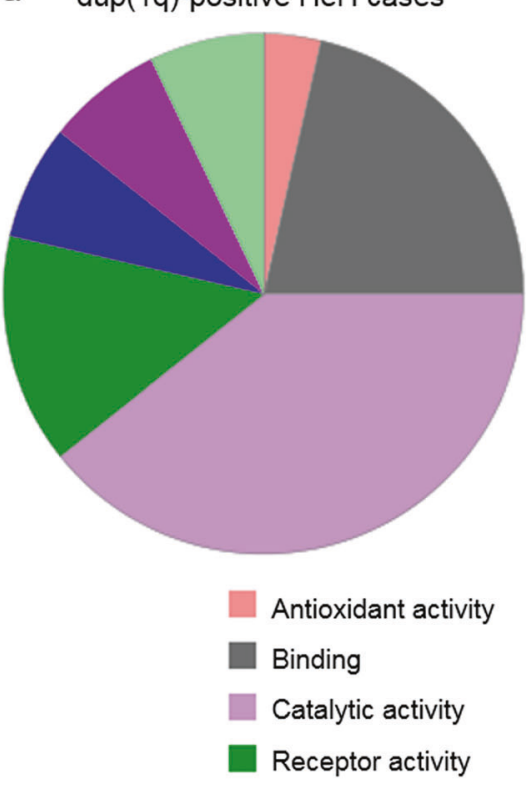

b dup(1q)-positive $t(1 ; 19)$ cases

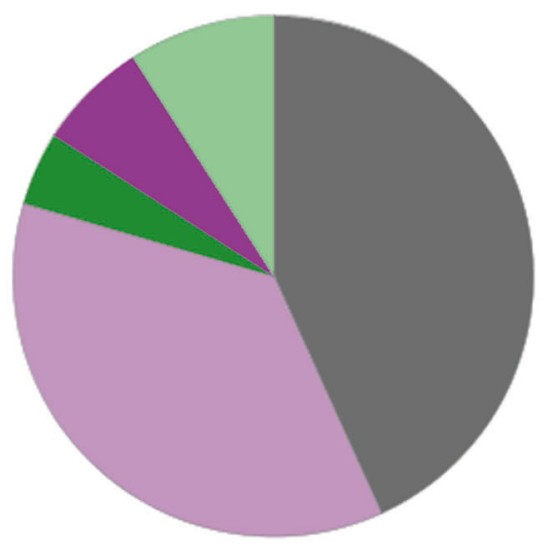

Signal transducer activity

Structural molecule activity

Transporter activity
Analyses of carcinomas of the breast, liver, and ovary and Wilms' tumors with 1q rearrangements have indicated that the near-centromeric $1 \mathrm{q}$ region is break-prone due to hypomethylation of sat II DNA[35, 41, 42]. However, we previously showed that sat II DNA on 1q is not hypomethylated in dup(1q)-positive $\mathrm{HeH}[21]$, strongly suggesting that other mechanisms underlie breaks in the nearcentromeric region of $1 \mathrm{q}$ in pediatric $\mathrm{BCP}$ ALL, for example palindromic low-copy repeats in the vicinity of the breakpoint cluster region, as previously identified on $17 \mathrm{p}$ in idic(17)(p11)-positive malignancies[43-45]. The genomic sequence spanning the centromere and proximal $1 \mathrm{q}$ is highly repetitive. This, unfortunately, prevented exact breakpoint designations by the SNP-A and targeted deep sequencing analyses of the dup(1q) breakpoints and made it impossible to ascertain the possible mechanistic role of the genomic architectural features surrounding the 1q21.1 breakpoints. However, in the few cases with more distal breakpoints, we were able to identify small deletions adjacent to the breakpoints. It is presently unknown, and methodologically difficult to address, whether these were present before, possibly increasing the susceptibility for, the $\operatorname{dup}(1 \mathrm{q})$ formation or occurred at the time of breakage or during the subsequent DNA repair steps.

It is generally assumed that gene-dosage effects affecting gene expression patterns are the pathogenetically important outcome of chromosomal gains. However, there are several examples of gains associated with gene mutations on the chromosomes involved, such as KIT mutations and trisomy 4 and internal tandem duplications of KMT2A and trisomy 11 in acute myeloid leukemia, MET mutations and trisomy 7 in hereditary papillary renal carcinomas, and JAK2 mutations and trisomy 9 in polycythemia vera[46-49]. In order to investigate whether also gains of $1 \mathrm{q}$ are associated with mutations of genes on this chromosome arm, we analyzed the breakpoint regions and all exons on 1q using deep sequencing on a discovery cohort of seven dup(1q)positive cases followed by TCA analyses of 111 cases w/wo 1q gains. Although non-synonymous somatic SNVs in the BLZF1, FMN2, KCNT2, LCE1C, NES, and PARP1 genes were identified in the cases with dup $(1 \mathrm{q})$, the TCA analysis of the validation cohort revealed similar SNV frequencies of these genes in cases w/wo gain of 1q. Furthermore, none of the mutated genes was differentially expressed in the RNASeq analysis (Supplementary Tables 7 and 8). Thus, we conclude that these SNVs were passengers, without any association with $\operatorname{dup}(1 \mathrm{q})$ as such.

We and others have previously identified altered methylation patterns in cases with genomic gains, such as hypomethylation of gene-poor regions on the trisomic/tetrasomic chromosomes in $\mathrm{HeH}$, on chromosomes 7 and 14 in colon cancer with trisomies of these chromosomes, on chromosome 8 in constitutional trisomy 8 mosaicism, and on $12 p$ in Pallister-Killian syndrome with gain of $i(12 p)$ [50-54]. We hence hypothesized that also gain of 1q might be associated with such methylation changes and ascertained the methylation status of cancer tissue-specific differentially methylated regions, promoters, $\mathrm{CpG}$ islands, shores, and shelves on 1q. However, HCA and PCA did not separate $\mathrm{HeH}$ and $\mathrm{t}(1 ; 19)$ cases w/wo dup(1q) cases based on 1q status (Supplementary Figure 4) and less than $1 \%$ of the $36,259 \mathrm{CpG}$ sites on 1q were differentially methylated. Thus, we found no evidence that dup(1q) is associated with any major methylation changes on $1 \mathrm{q}$. 
Only a handful of the differentially methylated CpGs were associated with gene expression changes in the cases analyzed by both BS-Seq and RNA-Seq. Interestingly, one of the genes hypomethylated and overexpressed in the dup (1q)-positive cases was $U C K 2$, located in 1q24.1 and coding for uridine-cytidine kinase 2 . This gene has previously been reported to be upregulated in a few dup(1q)-positive $\mathrm{HeH}$ cases[21], and overexpression of UCK2 was recently shown to correlate with progression/poor prognosis in breast cancer[55]. However, UCK2 was not among the close to 200 significantly overexpressed genes in the present series of HeH cases with 1q gain (Supplementary Table 7), making this gene an unlikely candidate to play an important pathogenetic or clinical role in $\mathrm{HeH}$.

The RNA-Seq analysis revealed that gain of $1 \mathrm{q}$ had a profound impact on both the global gene expression profiles and on the expression of genes on this chromosome arm (Fig. 1; Supplementary Tables 7 and 8). It is noteworthy that the deregulated genes in the dup(1q)-positive $\mathrm{HeH}$ and $\mathrm{t}$ $(1 ; 19)$ cases were, with only a few exceptions, distinct. Hence, the gene-dosage effects of 1q gains in pediatric BCP ALL are context dependent, varying among genetic subgroups, possibly reflecting the presence of different primary, leukemia-initiating genetic changes and/or distinct differentiation stages of the cells of origin. Because a total of $>100$ genes on 1q were overexpressed in the $\mathrm{HeH}$ and $t$ $(1 ; 19)$ cases with 1q gains, it is impossible to pinpoint a specific target gene. However, CKS1B, coding for the CDC28 protein kinase regulatory subunit $1 \mathrm{~B}$ and known to be overexpressed in multiple myeloma[17, 18], was clearly not overexpressed. Thus, overexpression of CSK1 is not the functionally important outcome of dup(1q) in $\mathrm{HeH}$ and $\mathrm{t}$ $(1 ; 19)$ cases, again emphasizing the variable gene-dosage effects of 1q gains in B-lineage malignancies. In our previous, smaller study of dup(1q)-positive $\mathrm{HeH}$, we observed upregulation of the DAP3 gene in 1q22 in a few cases[21]. This gene was also overexpressed in the present, larger cohort of $\mathrm{HeH}$ cases with 1q gain (Supplementary Table 7). DAP3 codes for the death-associated protein 3 that promotes apoptosis. This gene would hence not be expected to be upregulated in cancer; loss of function would be more likely. This notwithstanding, it is overexpressed in several types of malignancy, such as breast cancer, gastric cancer, thymoma, and glioblastoma[56-59]. Although overexpression of DAP3 may be pathogenetically important, we deem it too simplistic to reduce the functional consequence of dup(1q) in $\mathrm{HeH}$ to gain and deregulation of a single gene. In contrast, overexpression of multiple genes, affecting different pathways and cellular processes, may be the essential outcome.

Interestingly, although the deregulated genes in the dup (1q)-positive $\mathrm{HeH}$ and $\mathrm{t}(1 ; 19)$ cases were, with few exceptions, distinct (Supplementary Tables 7 and 8), GO analyses of the overexpressed genes on 1q revealed similar frequencies and types of molecular functions, biological processes, and protein classes of the upregulated genes in the two groups, with the majority encoding nucleic acid/ protein binding factors or proteins with catalytic activity (Fig. 2 and Supplementary Figures 7 and 8). In fact, the frequent overexpression of genes coding for nucleic acid binding proteins, such as transcription factors, may be one reason for the quite pronounced impact of dup(1q) on the global gene expression patterns in both $\mathrm{t}(1 ; 19)$ and $\mathrm{HeH}$ cases (Fig. 1).

In conclusion, dup(1q) in pediatric BCP ALL is not associated with SNVs or methylation changes on 1q. Instead, the pathogenetically important consequence of dup (1q) is a gene-dosage effect, and although the deregulated genes differ between dup(1q)-positive $\mathrm{HeH}$ and $\mathrm{t}(1 ; 19)$ cases, the overexpressed genes on 1q are associated with similar molecular functions, biological processes, and protein classes irrespective of genetic subtype.

Acknowledgements We thank the SciLifeLab core facilities and the National Genomic Infrastructure. This study was supported by grants from the Swedish Cancer Society (CAN 2017/291), the Swedish Childhood Cancer Foundation (PR2015-0006), the Swedish Research Council (2016-01084), Governmental Funding of Clinical Research within the National Health Service (2014/354), and the Royal Physiographic Society of Lund. The computations were performed on resources provided by SNIC through Uppsala Multidisciplinary Center for Advanced Computational Science (UPPMAX) under Project b2013219. Support by NBIS (National Bioinformatics Infrastructure Sweden) is gratefully acknowledged.

Funding The Swedish Cancer Society (CAN 2017/291), the Swedish Childhood Cancer Foundation (PR2015-0006), the Swedish Research Council (2016-01084), Governmental Funding of Clinical Research within the National Health Service (2014/354), and the Royal Physiographic Society of Lund.

Author's contributions RG planned and performed research and wrote the paper, SD performed the bioinformatic analysis and wrote the paper, AL and PW developed tools for bioinformatic analysis of BSseq data, LO, AB, HL, MR, KBL-S, KP, and TF performed research, AI planned research, and $\mathrm{BJ}$ planned research and wrote the paper. The paper was reviewed and approved by all the authors.

\section{Compliance with ethical standards}

Conflict of interest The authors declare that they have no conflict of interest.

Open Access This article is licensed under a Creative Commons Attribution 4.0 International License, which permits use, sharing, adaptation, distribution and reproduction in any medium or format, as long as you give appropriate credit to the original author(s) and the source, provide a link to the Creative Commons license, and indicate if changes were made. The images or other third party material in this article are included in the article's Creative Commons license, unless indicated otherwise in a credit line to the material. If material is not included in the article's Creative Commons license and your intended use is not permitted by statutory regulation or exceeds the permitted 
use, you will need to obtain permission directly from the copyright holder. To view a copy of this license, visit http://creativecommons. org/licenses/by/4.0/.

\section{References}

1. Johansson B, Mertens F, Mitelman F. Clinical and biological importance of cytogenetic abnormalities in childhood and adult acute lymphoblastic leukemia. Ann Med. 2004;36:492-503.

2. Mitelman F, Johansson B, Mertens F. Mitelman Database of Chromosome Aberrations and Gene Fusions in Cancer. http:// cgap.nci.nih.gov/Chromosomes/Mitelman 2017.

3. Mullighan CG, Goorha S, Radtke I, Miller CB, Coustan-Smith E, Dalton JD, et al. Genome-wide analysis of genetic alterations in acute lymphoblastic leukaemia. Nature. 2007;446:758-64.

4. Paulsson K, Forestier E, Lilljebjörn H, Heldrup J, Behrendtz M, Young BD, et al. Genetic landscape of high hyperdiploid childhood acute lymphoblastic leukemia. Proc Natl Acad Sci USA. 2010;107:21719-24.

5. Groupe Français de Cytogénétique Hématologique. Collaborative study of karyotypes in childhood acute lymphoblastic leukemias. Leukemia. 1993;7:10-19.

6. Johansson B, Mertens F, Mitelman F. Secondary chromosomal abnormalities in acute leukemias. Leukemia. 1994;8:953-62.

7. Raimondi SC, Pui CH, Hancock ML, Behm FG, Filatov L, Rivera GK. Heterogeneity of hyperdiploid (51-67) childhood acute lymphoblastic leukemia. Leukemia. 1996;10:213-24.

8. Chagtai T, Zill C, Dainese L, Wegert J, Savola S, Popov S, et al. Gain of $1 \mathrm{q}$ as a prognostic biomarker in Wilms tumors (WTs) treated with preoperative chemotherapy in the International Society of Paediatric Oncology (SIOP) WT 2001 Trial: A SIOP Renal Tumours Biology Consortium Study. J Clin Oncol. 2016;34:3195-203.

9. Garcia JL, Hernandez JM, Gutierrez NC, Flores T, Gonzalez D, Calasanz MJ, et al. Abnormalities on $1 \mathrm{q}$ and $7 \mathrm{q}$ are associated with poor outcome in sporadic Burkitt's lymphoma. A cytogenetic and comparative genomic hybridization study. Leukemia. 2003;17:2016-24.

10. Johansson B, Mertens F, Mitelman F. Cytogenetic evolution patterns in non-Hodgkin's lymphoma. Blood. 1995;86:3905-14.

11. Marzin Y, Jamet D, Douet-Guilbert N, Morel F, Le Bris MJ, Morice P, et al. Chromosome 1 abnormalities in multiple myeloma. Anticancer Res. 2006;26:953-9.

12. Mertens F, Johansson B, Höglund M, Mitelman F. Chromosomal imbalance maps of malignant solid tumors: a cytogenetic survey of 3185 neoplasms. Cancer Res. 1997;57:2765-80.

13. Nilsson T, Höglund M, Lenhoff S, Rylander L, Turesson I, Westin $\mathrm{J}$, et al. A pooled analysis of karyotypic patterns, breakpoints and imbalances in 783 cytogenetically abnormal multiple myelomas reveals frequently involved chromosome segments as well as significant age- and sex-related differences. $\mathrm{Br} \mathrm{J}$ Haematol. 2003;120:960-9.

14. Sawyer JR, Waldron JA, Jagannath S, Barlogie B. Cytogenetic findings in 200 patients with multiple myeloma. Cancer Genet Cytogenet. 1995;82:41-49.

15. Zudaire I, Odero MD, Caballero C, Valenti C, Martinez-Penuela $\mathrm{JM}$, Isola $\mathrm{J}$, et al. Genomic imbalances detected by comparative genomic hybridization are prognostic markers in invasive ductal breast carcinomas. Histopathology. 2002;40:547-55.

16. Ghose T, Lee CLY, Fernandez LA, Lee SHS, Raman R, Colp P. Role of 1q trisomy in tumorigenicity, growth, and metastasis of human leukemic B-cell clones in nude mice. Cancer Res. 1990;50:3737-42.

17. Fonseca R, Van Wier SA, Chng WJ, Ketterling R, Lacy MQ, Dispenzieri A, et al. Prognostic value of chromosome 1q21 gain by fluorescent in situ hybridization and increase $C K S 1 B$ expression in myeloma. Leukemia. 2006;20:2034-40.

18. Shaughnessy J. Amplification and overexpression of $C K S 1 B$ at chromosome band 1q21 is associated with reduced levels of $\mathrm{p} 27^{\mathrm{Kip} 1}$ and an aggressive clinical course in multiple myeloma. Hematology. 2005;10:117-26.

19. Durrbaum M, Storchova Z. Effects of aneuploidy on gene expression: implications for cancer. FEBS J. 2016;283:791-802.

20. Tang YC, Amon A. Gene copy-number alterations: a cost-benefit analysis. Cell. 2013;152:394-405.

21. Davidsson J, Andersson A, Paulsson K, Heidenblad M, Isaksson $\mathrm{M}$, Borg $\AA$, et al. Tiling resolution array comparative genomic hybridization, expression and methylation analyses of dup(1q) in Burkitt lymphomas and pediatric high hyperdiploid acute lymphoblastic leukemias reveal clustered near-centromeric breakpoints and overexpression of genes in 1q22-32.3. Hum Mol Genet. 2007;16:2215-25.

22. Olsson L, Castor A, Behrendtz M, Biloglav A, Forestier E, Paulsson K, et al. Deletions of IKZFI and SPREDI are associated with poor prognosis in a population-based series of pediatric B-cell precursor acute lymphoblastic leukemia diagnosed between 1992 and 2011. Leukemia. 2014;28:302-10. 2014, 28, 302-310

23. Li H, Durbin R. Fast and accurate short read alignment with Burrows-Wheeler transform. Bioinformatics. 2009;25: 1754-60.

24. Cibulskis K, Lawrence MS, Carter SL, Sivachenko A, Jaffe D, Sougnez C, et al. Sensitive detection of somatic point mutations in impure and heterogeneous cancer samples. Nat Biotechnol. 2013;31:213-9.

25. Wang $\mathrm{K}, \mathrm{Li} \mathrm{M}$, Hakonarson $\mathrm{H}$. ANNOVAR: functional annotation of genetic variants from high-throughput sequencing data. Nucleic Acids Res. 2010;38:e164-e164.

26. Layer RM, Chiang C, Quinlan AR, Hall IM. LUMPY: a probabilistic framework for structural variant discovery. Genome Biol. 2014;15:R84.

27. Chiang C, Layer RM, Faust GG, Lindberg MR, Rose DB, Garrison $\mathrm{EP}$, et al. SpeedSeq: ultra-fast personal genome analysis and interpretation. Nat Methods. 2015;12:966-8.

28. DePristo MA, Banks E, Poplin R, Garimella KV, Maguire JR, Hartl C, et al. A framework for variation discovery and genotyping using next-generation DNA sequencing data. Nat Genet. 2011;43:491-8.

29. McKenna A, Hanna M, Banks E, Sivachenko A, Cibulskis K, Kernytsky A, et al. The genome analysis toolkit: a MapReduce framework for analyzing next-generation DNA sequencing data. Genome Res. 2010;20:1297-303.

30. Van der Auwera GA, Carneiro MO, Hartl C, Poplin R, Del Angel G, Levy-Moonshine A, et al. From FastQ data to high confidence variant calls: the Genome analysis toolkit best practices pipeline. Curr Protoc Bioinforma. 2013;11:11.10. 1-11.10.33.

31. Krueger F, Andrews SR. Bismark: a flexible aligner and methylation caller for Bisulfite-Seq applications. Bioinformatics. 2011;27:1571-2.

32. Akalin A, Kormaksson M, Li S, Garrett-Bakelman FE, Figueroa ME, Melnick A, et al. methylKit: a comprehensive R package for the analysis of genome-wide DNA methylation profiles. Genome Biol. 2012;13:R87.

33. Lilljebjörn H, Henningsson R, Hyrenius-Wittsten A, Olsson L, Orsmark-Pietras C, von Palffy S, et al. Identification of ETV6$R U N X 1$-like and DUX4-rearranged subtypes in paediatric B-cell precursor acute lymphoblastic leukaemia. Nat Commun. 2016;7:11790.

34. Brito-Babapulle V, Atkin NB. Break points in chromosome \#1 abnormalities of 218 human neoplasms. Cancer Genet Cytogenet. 1981;4:215-25. 
35. Narayan A, Ji W, Zhang XY, Marrogi A, Graff JR, Baylin SB, et al. Hypomethylation of pericentromeric DNA in breast adenocarcinomas. Int J Cancer. 1998;77:833-8.

36. Busson-Le Coniat M, Salomon-Nguyen F, Dastugue N, Maarek O, Lafage-Pochitaloff M, Mozziconacci MJ, et al. Fluorescence in situ hybridization analysis of chromosome 1 abnormalities in hematopoietic disorders: rearrangements of DNA satellite II and new recurrent translocations. Leukemia. 1999;13:1975-81.

37. Le Baccon P, Leroux D, Dascalescu C, Duley S, Marais D, Esmenjaud E, et al. Novel evidence of a role for chromosome 1 pericentric heterochromatin in the pathogenesis of B-cell lymphoma and multiple myeloma. Genes Chromosomes Cancer. 2001;32:250-64.

38. Itoyama T, Nanjungud G, Chen W, Dyomin VG, Teruya-Feldstein J, Jhanwar SC, et al. Molecular cytogenetic analysis of genomic instability at the 1q12-22 chromosomal site in B-cell non-Hodgkin lymphoma. Genes Chromosomes Cancer. 2002;35:318-28.

39. Wong N, Chan A, Lee SW, Lam E, To KF, Lai PBS, et al. Positional mapping for amplified DNA sequences on 1q21-q22 in hepatocellular carcinoma indicates candidate genes overexpression. J Hepatol. 2003;38:298-306.

40. Tomlinson GE, Douglass EC, Pollock BH, Finegold MJ, Schneider NR. Cytogenetic evaluation of a large series of hepatoblastomas: numerical abnormalities with recurring aberrations involving 1q12-q21. Genes Chromosomes Cancer. 2005;44: 177-84.

41. Ehrlich M, Hopkins NE, Jiang G, Dome JS, Yu MC, Woods CB, et al. Satellite DNA hypomethylation in karyotyped Wilms tumors. Cancer Genet Cytogenet. 2003;141:97-105.

42. Widschwendter M, Jiang G, Woods C, Muller HM, Fiegl H, Goebel G, et al. DNA hypomethylation and ovarian cancer biology. Cancer Res. 2004;64:4472-80.

43. Scheurlen WG, Schwabe GC, Seranski P, Joos S, Harbott J, Metzke S, et al. Mapping of the breakpoints on the short arm of chromosome 17 in neoplasms with an i(17q). Genes Chromosomes Cancer. 1999;25:230-40.

44. Barbouti A, Stankiewicz P, Nusbaum C, Cuomo C, Cook A, Höglund $\mathrm{M}$, et al. The breakpoint region of the most common isochromosome, $\mathrm{i}(17 \mathrm{q})$, in human neoplasia is characterized by a complex genomic architecture with large, palindromic, low-copy repeats. Am J Hum Genet. 2004;74:1-10.

45. Bien-Willner GA, Stankiewicz P, Lupski JR, Northup JK, Velagaleti GVN. Interphase FISH screening for the LCR-mediated common rearrangement of isochromosome $17 \mathrm{q}$ in primary myelofibrosis. Am J Hematol. 2005;79:309-13.

46. Caligiuri MA, Strout MP, Schichman SA, Mrozek K, Arthur DC, Herzig GP, et al. Partial tandem duplication of $A L L 1$ as a recurrent molecular defect in acute myeloid leukemia with trisomy 11 . Cancer Res. 1996;56:1418-25.
47. Zhuang Z, Park WS, Pack S, Schmidt L, Vortmeyer AO, Pak E, et al. Trisomy 7-harbouring non-random duplication of the mutant MET allele in hereditary papillary renal carcinomas. Nat Genet. 1998;20:66-69.

48. Beghini A, Ripamonti CB, Cairoli R, Cazzaniga G, Colapietro P, Elice F, et al. KIT activating mutations: incidence in adult and pediatric acute myeloid leukemia, and identification of an internal tandem duplication. Haematologica. 2004;89:920-5.

49. James C, Ugo V, Le Couedic JP, Staerk J, Delhommeau F, Lacout $\mathrm{C}$, et al. A unique clonal JAK2 mutation leading to constitutive signalling causes polycythaemia vera. Nature. 2005;434:1144-8.

50. Weber M, Davies JJ, Wittig D, Oakeley EJ, Haase M, Lam WL, et al. Chromosome-wide and promoter-specific analyses identify sites of differential DNA methylation in normal and transformed human cells. Nat Genet. 2005;37:853-62.

51. Davidsson J, Lilljebjörn H, Andersson A, Veerla S, Heldrup J, Behrendtz M, et al. The DNA methylome of pediatric acute lymphoblastic leukemia. Hum Mol Genet. 2009;18:4054-65.

52. Davidsson J, Veerla S, Johansson B. Constitutional trisomy 8 mosaicism as a model for epigenetic studies of aneuploidy. Epigenetics Chromatin. 2013;6:18.

53. Nordlund J, Backlin CL, Wahlberg P, Busche S, Berglund EC, Eloranta ML, et al. Genome-wide signatures of differential DNA methylation in pediatric acute lymphoblastic leukemia. Genome Biol. 2013;14:r105.

54. Davidsson J, Johansson B. Methylation and expression analyses of Pallister-Killian syndrome reveal partial dosage compensation of tetrasomy $12 \mathrm{p}$ and hypomethylation of gene-poor regions on 12-204p. Epigenetics. 2016;11:194-204.

55. Shen G, He P, Mao Y, Li P, Luh F, Ding G, et al. Overexpression of uridine-cytidine kinase 2 correlates with breast cancer progression and poor prognosis. J Breast Cancer. 2017;20:132-41.

56. Mariani L, Beaudry C, McDonough WS, Hoelzinger DB, Kaczmarek E, Ponce F, et al. Death-associated protein 3 (Dap-3) is overexpressed in invasive glioblastoma cells in vivo and in glioma cell lines with induced motility phenotype in vitro. Clin Cancer Res. 2001;7:2480-9.

57. Sasaki H, Ide N, Yukiue H, Kobayashi Y, Fukai I, Yamakawa Y, et al. Arg and DAP3 expression was correlated with human thymoma stage. Clin Exp Metastas-. 2004;21:507-13.

58. Wazir U, Jiang WG, Sharma AK, Mokbel K. The mRNA expression of DAP3 in human breast cancer: correlation with clinicopathological parameters. Anticancer Res. 2012;32:671-4.

59. Jia Y, Ye L, Ji K, Zhang L, Hargest R, Ji J, et al. Death-associated protein-3, DAP-3, correlates with preoperative chemotherapy effectiveness and prognosis of gastric cancer patients following perioperative chemotherapy and radical gastrectomy. Br J Cancer. 2014;110:421-9. 